\title{
El trasplante cardiaco por enfermedad de Chagas en México
}

Felipe Lozano-Kasten 1, Margarita Soto-Gutiérrez 1, Ezequiel Magallón-Gastélum 1, Marina KastenMonges ${ }^{1}$, Francisco Hinojosa-Alarcón ${ }^{2}$, Alejandro Aaron Peregrina-Lucano ${ }^{3}$

${ }^{11}$ Instituto Regional de Investigación en Salud Publica. CUCS. Universidad de Guadalajara, México, ${ }^{2}$ Servicio de Cardiología, CMNO, IMSS, ${ }^{3}$ Departamento de Farmacobiologia. CUCEI. Universidad de Guadalajara

\section{RESUMEN}

El objetivo de este escrito, es presentar los datos clínicos, quirúrgicos, paraclínicos, terapeúticos, y epidemiológicos que sustentan la existencia del primer caso de trasplante cardíaco por cardiopatía chagásica en México. Se trata de un paciente masculino de 35 años de edad, al que se realizó trasplante cardiaco por cardiomiopatia dilatada no compatible con la vida; comprobado por serología y aislamiento del $T$. cruzi posterior a la intervención quirúrgica realizada al presentar complicaciones posteriores al trasplante. A 9 años del trasplante, el paciente se considera curado y reintegrado al trabajo. Los pacientes diagnosticados clínicamente con miocardiopatía dilatada y serología positiva a $T$ cruzi, son frecuentes, en el orden de $15 \%$ en el país. La presencia del presente caso y la circunstancia en que se diagnosticó, orienta la existencia de un mayor número de casos ya trasplantados $\mathrm{y}$ no diagnosticados en el país. Por lo tanto, es necesario reconocer en pacientes candidatos a trasplante cardiaco por miocardiopatía dilatada, la enfermedad de Chagas.

Palabras clave: trasplante cardíaco, enfermedad de Chagas, México

\section{ABSTRACT \\ Hearth transplants for chagas disease in Mexico}

The aim of this report is to present the clinical, surgical, paraclinical, therapeutical and epidemiological data supporting the first heart transplant case related to Chagas disease in Mexico. The subject, a 35 year old male, was diagnosed with a dilated cardiomyopathy unable to support life. Serological testing was completed, and $T$. cruzi isolated after a surgical intervention due to post operatory complications to the transplant. Nine years after transplantation, the patient was considered cured and re-entered the labor force. Patients clinically diagnosed with dilated cardiomyopathy and positive serology for T. cruzi, are frequent, representing $15 \%$ in the country. The presence of this case and its diagnostic circumstances indicates the existence of a greater number of already transplanted undiagnosed cases in the country. Therefore it is necessary to recognize the role of Chagas disease in heart transplantation candidates due to dilated cardiomyopathy.

Key words: heart transplantation, chagas, Mexico.

\section{INTRODUCCIÓN}

El trasplante cardiaco por miocardiopatía dilatada por enfermedad de Chagas, es una entidad clínica-quirúrgica no documentada en México. El objetivo de la presente comunicación, es presentar las evidencias clínico-quirúrgicas, paraclínicas y

Autor para correspondencia: Felipe Lozano-Kasten, Justo Sierra 1940, Colonia Americana, Guadalajara, Jalisco. CP E-mail: f_lozano_k@hotmail.com Recibido: el 5 de septiembre de 2014. Aceptado para publicación: el 5 de enero de 2015 
Lozano-Kasten et al.

epidemiológicas de la existencia del primer caso de trasplante cardíaco por cardiopatía chagásica. La enfermedad de Chagas, es conocida en el país desde 1936 y los datos sobre seroprevalencia se han consolidado y se estiman en cerca de $1.6 \%$ en la población del país (1); manteniéndose en niveles relativamente altos en algunas regiones. La cardiomiopatía chagásica crónica podría estar afectando a individuos con enfermedad cardiaca, aún cuando los casos no se reportan debido a la ausencia de especificidad del cuadro clínico (2). Diversos estudios muestran que la cardiopatía chagásica crónica en México es una causa común de la miocardiopatía dilatada, debido a su distribución regional y merece atención (1). En el Estado de Campeche, se observa serología positiva para Tripanosoma cruzi en pacientes con diagnóstico clínico de miocardiopatía dilatada del orden del 15\% (3).

Actualmente, entre las principales causas mundiales por las que se indica el trasplante de corazón están las siguientes: la cardiomiopatía asociada a isquemia (42\%); miocardiopatía dilatada (33\%); enfermedades valvulares y otros trastornos (25\%) (4).

En el mundo hay miles de personas a las que se ha trasplantado un corazón por enfermedad de Chagas y los casos reportados han sido numerosos. El primer caso de trasplante cardiaco en América Latina y tercero realizado en el mundo fue realizado en 1968 en Brasil, por el Dr. Zerbini y Decourt, precisamente, en un paciente con miocardiopatía dilatada por enfermedad de Chagas (5). En Estados Unidos de Norteamérica se reportó el primer caso de trasplante cardíaco por miocardiopatía dilatada por enfermedad de Chagas en 2006 y entre 2006 y 2012 han sido 11 las personas ya sometidas a trasplante por este motivo (6).

Presentación del caso clínico. Se trata de un paciente del sexo masculino de 35 años (JMPJ), que nació y vive actualmente en el municipio de Tlajomulco de Zúñiga, Estado de Jalisco, México. Como antecedente heredo-familiar importante se señala que su madre es seropositiva a T.cruzi. La vivienda con carencias físicas y ambientales, convivía con animales de corral y domésticos desde la infancia, obrero de una fábrica local de dulces, con antecedentes de tabaquismo por 14 años y 6 años de alcoholismo; nivel socioeconómico, bajo y estado civil, casado con tres hijos. Se diagnosticó cardiópata en el año 2003, llegando a presentar disfunción biventricular severa, con múltiples ingresos a hospitales debido a insuficiencia cardiaca en clase funcional IV. En el ultimo ingreso, se documentó expectoración hemoptoica por hipertensión venocapilar severa. El tratamiento médico fue a base de digitalicos, diuréticos, antialdosteronicos y anticoagulantes. En la exploración física: Talla 1.65 m., Peso 53 kg., con hipotrofia muscular generalizada, tinte ictérico en ambas conjuntivas, ingurgitación yugular de 4 a $5 \mathrm{~cm}$, TA 95/50 mm $\mathrm{Hg}, \mathrm{Fc} 80 \mathrm{x}$ 1', superficie corporal de 1:65 m2. Campos pulmonares con leve hiperventilación en base izquierda. Soplo sistólico mitral, soplo sistólico tricuspídeo, componente pulmonar del segundo ruido ++ , hepatomegalia congestiva de $8 \mathrm{~cm}$, sin edema periférico. Paraclínicos (23/05/05); Rx de Torax: Cardiomegalia grado III sin patología pulmonar, ECG: RS, CAI; BRIHH; CVI, PR de 20 ms. ECO Trans Torácico: AI setenta y uno por sesenta y seis, AD setenta por sesenta y seis. VD treinta y nueve. VI DDVI $84 \mathrm{~mm}$, DSVI 74mm, se calculó una FE de 15\% por Simpson y $23 \%$ por Teichholz. Insuficiencia mitral moderada, secundaria a dilatación de anillo mitral $(35 \mathrm{~mm})$. Insuficiencia tricuspídea moderada que permitió calcular una presión sistólica pulmonar de $46 \mathrm{mmHg}$. Dilatación del anillo tricuspídeo a $34 \mathrm{~mm}$. insuficiencia aortica leve. Hb 15 g/dL., Hto. 47\%, leucocitos 10,100, plaquetas 128,000, glucosa $79 \mathrm{mg} / \mathrm{dL}$, urea 68 $\mathrm{mg} / \mathrm{dL}$, creatinina $1.1 \mathrm{mg} / \mathrm{dL}$, ácido úrico $6.6 \mathrm{mg}$ / $\mathrm{dL}$, grupo sanguíneo $\mathrm{O} \mathrm{Rh}$ positivo, colesterol

\section{Revista Biomédica}


Trasplante cardíaco por enfermedad de Chagas en México

total $60 \mathrm{mg} / \mathrm{dL}$, TG $82 \mathrm{mg} / \mathrm{dL}$, lipoproteina de baja densidad $34 \mathrm{mg} / \mathrm{dL}$, lipoproteina de alta densidad $8 \mathrm{mg} / \mathrm{dL}, \mathrm{BT} 8.5 \mathrm{mg} / \mathrm{dL}$, BD $5.2 \mathrm{mg} /$ $\mathrm{dL}$, BI $3.3 \mathrm{mg} / \mathrm{dL}$, TP 14/11, seg, INR 1.32, TPT 42/33 seg, perfil Viral: Hepatitis B y C (-), HIV (-) VDRL ( - ), anticuerpos anti toxoplasma IgG (+) 151, IgM (-), Antígeno Anti Citomegalovirus (+), IgG 133, IgM (-), Anticuerpos Anti rubeola $\operatorname{IgM}(+)$ 43., IgM (-) serología positiva a $T$ cruzi; en el año 2003 Hemaglutinación Indirecta (HAI) 1:16, ELISA $(+)$ en el año 2004 , HAI 1:8., $\operatorname{ELISA}(+)$.

Con todos los datos anteriores, los médicos tratantes concluyeron que se trataba de un paciente con una miocardiopatía dilatada muy posiblemente idiopática, con cardiomegalia III, HVCP severa, HAP moderada, en clase funcional IV NYHA. Resistencias pulmonares totales 3.2 unidades Woods, cuyo pronostico es mortal y se le propuso trasplante cardiaco ortotópico.

Por el antecedente de las pruebas positivas a $T$. cruzi relizadas en su centro de atención primaria comunitario (HAI y ELISA) en 2003 y 2004, ameritó interconsulta al servicio de infectología, definiendo que, de corroborarse la enfermedad de Chagas, no se consideraría candidato para trasplante cardiaco, por la posibilidad de reactivación de la enfermedad por inmunosupresión y afectación al órgano trasplantado. Se realizó una prueba de gota gruesa y tres biopsias endomiocárdiacas intentando observar al parasito resultando todas negativas, por lo cual permitió avanzar en el protocolo de trasplante cardiaco. El trasplante se realizó en agosto del 2005; el corazón se obtuvo de un joven de 22 años víctima de muerte cerebral por accidente.

En el postoperatorio tardío, presentó una pancreatitis que le ocasionó como complicación diabetes mellitus. A los 6 meses del trasplante, y con una evolución tórpida respecto al control de la diabetes mellitus, el cardiólogo revalúa el caso, y considerando la serología positiva a $T$. cruzi de los años 2003 y 2004, se plantea la posibilidad de una reactivación de la infección chagásica por el manejo con inmunosupresores. Se realizan nuevos examenes serológicos y xenodiagnóstico, resultando HAI 1:32, Elisa positiva, Elisa recombinante positiva, xenodiagnóstico positivo (Cuadro No. 1).

Con estos resultados (Xenodiagnostico positivo) el paciente se diagnóstico en una fase aguda de la Enfermedad. Se administró tratamiento con Benznidazol (N-benzil-2-nitro-

Cuadro 1

Resultados de la serología y xenodiagnóstico

\begin{tabular}{cccc}
\hline Fecha & HAI & ELISA & $\begin{array}{c}\text { ELISA } \\
\text { Recombinante }\end{array}$ \\
\hline $16 / 10 / 2003$ & Negativo & Negativo & No realizado \\
$26 / 11 / 2003$ & $1: 16$ & Positiva & No realizado \\
$19 / 05 / 2004$ & $1: 8$ & Positiva & No realizado \\
$15 / 02 / 2006$ & $1: 32$ & Positiva & Positiva \\
$10 / 03 / 2006$ & Xenodiagnóstico $(+) 15 / 04 / 2006$ & \\
\hline
\end{tabular}

imidazolacetidamida) para actúar sobre los tripomastigotes circulantes y los amastigotes intracelulares (7). Se utilizaron concentraciones inhibitorias de Benznidazol a niveles séricos de $3-8 \mu \mathrm{g} / \mathrm{ml}$, a dosis de $5-7 \mathrm{mg}$ x $\mathrm{kg}$ de peso $\mathrm{x} 30$ días, monitorizándose dicha concentración sérica de Benznidazol, mediante el método analítico de cromatografía liquida de alta resolución con detección ultravioleta para determinar los niveles terapéuticos inhibitorios del T. cruzi durante las 4 semanas que duro el tratamiento (Cuadro No. 2). El paciente no presento reacción adversa al fármaco y fue dado de alta 12 meses después del tratamiento con el Benznidazol. A nueve años del trasplante, el paciente se encuentra sin evidencia de complicaciones, con una calidad de vida que le ha permitido regresar y ser aceptado nuevamente en la misma empresa en que trabajaba anteriormente, y convivir con su familia. 
Lozano-Kasten et al.

\section{DISCUSIÓN}

El caso muestra la evidente incertidumbre clínica que existe sobre la miocardiopatía dilatada de origen chagásico entre los grupos médicos que realizan trasplantes actualmente en nuestro país. La incertidumbre principal para entrar al protocolo de trasplante fue que el paciente estuviera infectado por T. Cruzi. La biopsia miocárdica se utilizó como parámetro de infección y lesión cardiaca, tratando de observar el parásito; al no evidenciarse por esta técnica, se procedió al trasplante. La biopsia endomiocárdica en enfermedad de Chagas permite estudiar los aspectos histológicos, ultraestructurales, histoquímicos e inmunohistoquímicos así como la fibrosis, hipertrofia celular, necrosis, alteraciones degenerativas focales e infiltrado inflamatorio; sin embargo, los nidos de parásitos son prácticamente inaccesibles por este medio (8). El paciente fue trasplantado precisamente por desconocer el valor de la biopsia miocárdica en la miocarditis chagásica. Por otro lado, en los protocolos oficiales que regulan los procesos de la donación, hasta el trasplante de órganos, tejidos y células, en ningún momento se menciona la infección por Tripanosoma cruzi como contraindicación o indicación de trasplante cardiaco. Simplemente, no existe la enfermedad en el protocolo del Consejo Nacional de Trasplantes (9).

Las complicaciones en el paciente fueron las que finalmente permitieron revalorar el caso respecto a la infección por T. Cruzi y, por tanto, del origen Chagásico. La confirmación generó la posibilidad del tratamiento y la evolución del

\section{Cuadro 2}

Monitorización del Benzonidazol sérico durante el tratamiento

\begin{tabular}{cc}
\hline No. de muestras y fecha & $\begin{array}{c}\text { Concentración del } \\
\text { Benznidazol sérico } \mu \mathrm{g} / \mathrm{mL}\end{array}$ \\
\hline $1 / 12 / 05 / 08)$ & 8.15 \\
$2(19 / 05 / 06)$ & 10.14 \\
$3(29 / 05 / 06)$ & 7.91 \\
$4(05 / 06 / 06)$ & 9.37 \\
\hline
\end{tabular}

paciente y, seguramente, el éxito del trasplante. El presente caso muestra que el origen chagásico de la cardiopatía debe ser investigado ante la sospecha clínica en toda miocardiopatía dilatada en nuestro país y, asi, evitar en lo posible casos como el descrito y para adecuar el cuidado postoperatorio inmediato, mediato y tardío sobre todo, lo concerniente a la inmunosupresión. En los años 80, durante los primeros trasplantes de corazón debido a cardiomiopatía por Chagas efectuados en Brasil, se consideró un gran problema; sin embargo, hoy con nuevos y mejores inmunosupresores el problema es menor como lo demuestra el manejo posterior del paciente (1013).

\section{CONCLUSION}

La fragmentación y el escaso conocimiento sobre la miocardiopatía chagásica y el trasplante de corazón observado en este caso, plantea necesariamente la construcción de instrumentos validos y confiables para la identificación y manejo de la enfermedad de Chagas tanto en su fase aguda, crónica y de intervención quirúrgica. Entre los año de 1988 a 2013 (26 años) han sido 394 los trasplantes cardiacos registrados en México. (Figura 1) (14). No se tiene evidencia en la literatura nacional del origen chagásico en alguno de ellos. Esta información puede inducir a

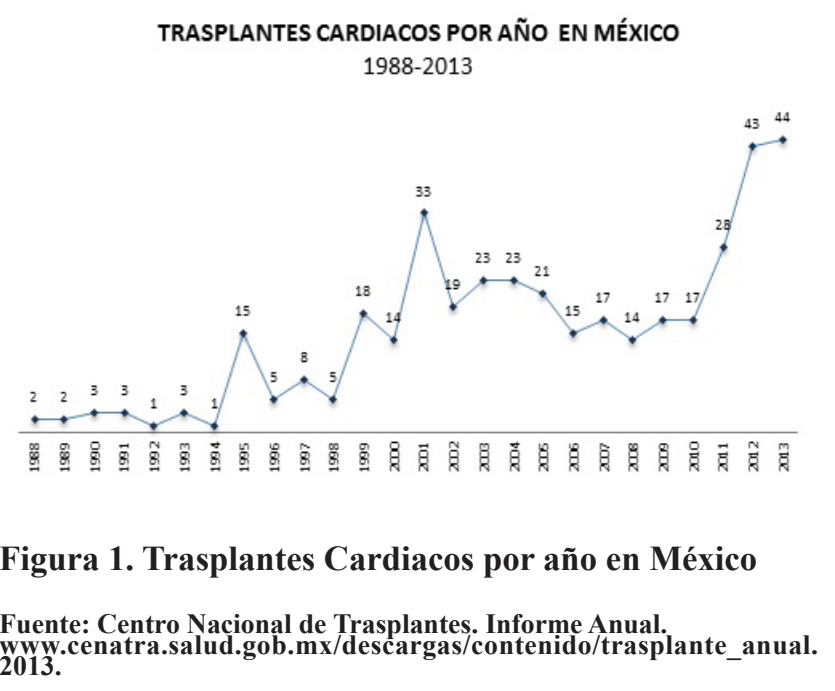

\section{Revista Biomédica}


Trasplante cardíaco por enfermedad de Chagas en México

los tomadores de decisiones en salud a no mejorar o invertir en la adquisición de aptitudes por parte de los médicos para diagnosticar la enfermedad tanto en las curriculas universitarias como en el Sector Salud. Si se considera que en el país existen más de un millón de personas infectadas y en el Estado de Campeche, existe una serología positiva a $T$. cruzi en pacientes con diagnóstico clínico de miocardiopatía dilatada del orden del 15\% (3), entonces quizá de los 394 pacientes trasplantados podríamos suponer que 59 casos pudieran haber sido por cardiopatía chagásica. Lo anterior es una oportunidad para mejorar las aptitudes de los clínicos, y la sobrevivencia de pacientes con esta enfermedad.

\section{REFERENCIAS}

1. Sierra-Johnson J, Olivera-Mar A, Monteón-Padilla Victor M, Reyes Pedro A, Vallejo M. Panorama epidemiológico y clínico de la cardiopatía chagásica en México. Rev. Saúde Pública. 2005; 39 (5): 754-60.

2. Dumonteil E. Update on Chagas disease in Mexico. Salud Pública Méx 1999; 41: 322-327.

3. Alducin-Téllez, César et al. Prevalencia de serología positiva para Trypanosoma cruzi en pacientes con diagnóstico clínico de miocardiopatía dilatada en el Estado de Campeche, México. Arch. Cardiol. Méx., 2011; 81 (3): 204-207.

4. Martínez Gutiérrez, E. et al. Experiencia con trasplante cardiaco: Hospital General Centro Médico Nacional "La Raza", IMSS. Cirujano General. 2002; 24 (2): 140-143.
5. Fiorelli AI, et al. Trasplante cardiaco en pacientes con enfermedad de Chagas. Experiencia de un único centro. Cirugía Cardiovascular. 2011; 18(3): 225-232.

6. Kransdorf EP, et al. Heart transplantation for Chagas cardiomyopathy in the United States. Am J Transplant. 2013; 13(12):3262-3268.

7. Cerisola JA. Chemotherapy of Chagas infection in man. Scientific Publication, PAHO 1977; N 347.

8. Mota JD, Suárez C, García J. Biopsia endomiocárdica en miocarditis chagásiga e inespecífica. Gac Méd Caracas 1998;106(3):358-368.

9. Secretaría de Salud, Reglamento del Consejo Nacional de Trasplantes http://www.cenatra.salud. gob.mx/interior/sistema_nacional_consejo_nacional. html. 2014.

10. Fiorelli AI, Santos RH, Oliveira JL Jr, LourençoFilho DD, Dias RR, Oliveira AS, et al. Hearth transplantation in 107 cases of Chagas' disease. Transplant Proc. Jan-Feb. 2011;43(1):220-4

11. Bocchi EA, Bellotti G, Mocelin AO, et al. Heart transplantation for cronic Chagas' heart DISEASE. Ann Thorac Surg. Jun 1996;61:1727-33.

12. Campos SV, Strabelli TM, Amato Neto V, et al. Risk factors for Chagas' disease reactivation after heart transplantation. J. Heart Lung Transplant. Jun 2008;27(6):597-602.

13. Bacal F, Silva CP, Pires PV, Mangini S, Fiorelli AI, Stolf NG, et al. Transplantation for Cagaas'disease: an overview of immunosuppression and reactivación in the last two decades. Clinc Transplant. Mar-Apr 2010;24(2):29-34.

14. Secretaría de Salud. Centro Nacional de Trasplantes. Informe Anual 2013. Disponible en www.salud.gob. mx/unidades/cdi/nom/compi/ReinCNT.html 